Revue d'histoire de l'Amérique française

REVUE D.HISTOIRE DE L'AMÉRIQUE FRANÇAISE

\title{
Index par auteur de compte rendu, note critique ou note bibliographique
}

Index, 1987

Index des volumes XXXI-XL (juin 1977 - printemps 1987)

URI : https://id.erudit.org/iderudit/305687ar

DOI : https://doi.org/10.7202/305687ar

Aller au sommaire du numéro

Éditeur(s)

Institut d'histoire de l'Amérique française

\section{ISSN}

0035-2357 (imprimé)

1492-1383 (numérique)

Découvrir la revue

Citer ce document

(1987). Index par auteur de compte rendu, note critique ou note bibliographique. Revue d'histoire de l'Amérique française, 117-136. https://doi.org/10.7202/305687ar d'utilisation que vous pouvez consulter en ligne.

https://apropos.erudit.org/fr/usagers/politique-dutilisation/ 


\section{Index des comptes rendus,} notes critiques et notes bibliographiques par auteur de compte rendu, note critique ou note bibliographique 
(NC) indique une note critique

\section{A}

ABENON, Lucien René, Jacques MATHIEU, Le commerce entre la Nouvelle-France et les Antilles au XVIIIe siècle, 37, 1: 111-114; Pierre PLUCHON, Histoire des médecins et pharmaciens de Marine et des colonies, 40 , 2: 302-303; Robert Louis STEIN, LégerFélicité Sonthonax, the Lost Sentinel of the Republic, 40, 2: 290-293

ALLAIRE, Gratien, Claude CHAMPAGNE, Les débuts de la mission dans le Nord-Ouest canadien. Mission et Église chez Mgr Vital Grandin, o.m.i. (1829-1902), 40, 1: 114115

ANCTIL, Pierre, N. H. BEDFORD, Anthologie de la littérature franco-américaine de la Nouvelle-Angleterre, 35, 2: 270-273; Raymond BRETON et Pierre SAVARD, eds., The Quebec and Acadian Diaspora in North America, 37, 2: 325-327

ARĖ, Richard, Michel BRUNET, Notre passé, le présent et nous, 31, 1:85-88

ARMSTRONG, Robert, John McCALLUM, Unequal Beginnings: Agriculture and Economic Development in Quebec and Ontario until 1870, 35, 2: 281-284 et note rectificative, 35, 3: 407; Richard POMFRET, The Economic Development of Canada, 37, 3: 470-472; Yves ROBY, Les Québécois et les investissements américains (1918-1929), 32, 1: 105-111

AXELROD, Paul, Stanley Brice FROST, McGill University: For the Advancement of Learning, vol. II: 1895-1971, 39, 2: 284286

B

BAILLARGEON, Denyse, Geneviève AUGER et Raymonde LAMOTHE, De la poêle à frire à la ligne de feu - La vie quotidienne des Québécoises pendant la guerre 39-45, 38, 1: 95-97; W. J. C. CHERWINSKI et Gregory S.KEALEY, eds., Lectures in Canadian Labour and Working-Class History, 40, 3: 434-436; Institut québécois de recherche sur la culture, Identités féminines. Mémoire et création, 40, 4: 602-605

BEAUCAGE, Benoît, René HARDY et al., L'exploitation forestière en Mauricie. Dossier statistique, 1850-1930, 36, 4: 589-591; René HARDY et al., La Mauricie et les Bois-Francs. Chronologie, 1850-1950, 36, 4: 589-591; Jean ROY et al., Les populations municipales et paroissiales de la Mauricie. Dossier statistique, 1850-1971, 36, 4: 589-591; Normand SEGUIN et al., L'agriculture en Mauricie. Dossier statistique, 1850-1950, 36, 4: 589-591; Louise VERREAULT-ROY, Répertoire cartographique de la Mauricie, 1800-1950, 36, 4: 589-591

BEAUDET, Céline, B. JULIEN, H. BEAUCHAMP-RANK et Paul WYCZYNSKI, Le théâtre canadien-français, 31, 2: 275-276

BEAUREGARD, Ludger, Louis-Edmond HAMELIN, Nordicité canadienne, 31, 1: 98-100

BEHIELS, Michael D., Paul-André COMEAU, Le Bloc populaire 1942-1948, 37, 2: 331334; Georges-Henri LÉVESQUE, Souvenances I. Entretiens avec Simon Jutras, 38, 4: 602-604

BÉLANGER, Jules, Anselme CHIASSON, Les iles de la Madeleine. Vie matérielle et sociale, 37, 1: 95-97

BÉLANGER, Réal, Paul BERNIER, Ernest Lapointe, député de Kamouraska, 1904 1919, 33, 3: 463-465; Lionel FORTIN, Félix-Gabriel Marchand, 34, 1: 112-114

BERGERON, Claude, Mathilde BROSSEAU, Le style néo-gothique dans l'architecture au Canada, 37, 2: 327-329; Christina CAMERON et Janet WRIGHT, Le style second Empire dans l'architecture canadienne, 37, 2: 327-329

BERGEVIN, Hélène, J. VEILLETTE, G. WHITE et al., Early Indian Village Churches: Wooden Frontier Architecture in British Columbia, 33, 1: 100-101

BERNARD, André, Vincent LEMIEUX, Personnel et partis politiques au Québec. Aspects historiques, 36, 3: 435-436

BERNARD, Jean-Paul, André LAVOIE, dir., Répertoire des parlementaires québécois, 
1867-1978, 35, 3: 437; Denis MONIÈRE et André VACHET, Les idéologies au Québec. Bibliographie, 35, 3: 438; D. A. MUISE, ed., A Reader's Guide to Canadian History, 1: Beginnings to Confederation, 37, 3: 467-468; Gérard PARIZEAU, Les Dessaulles, seigneurs de Saint-Hyacinthe Chronique maskoutaine du XIXe siècle, 33, 2: 272-273; Maurice RABOTIN, Le vocabulaire politique et socio-ethnique à Montréal de 1839 à 1842, 32, 2: 270-272; JeanPaul ROULEAU et Paul STRYCKMAN, dir., Sciences sociales et églises. Questions sur l'évolution religieuse du Québec, 35, 3: 428-430; Elinor Kyte SENIOR, Redcoats and Patriotes: The Rebellions in Lower Canada, 1837-1838, 40, 1: 113-114; UNIVERSITÉ DU QUÉBEC À MONTRÉAL, Inventaire des brochures conservées au Service des archives, 1771-1967, 32, 4: 644; Louise VOYER, Saint-Hyacinthe. De la seigneurie à la ville québécoise, 35, 3: 434

BERNIER, Jacques, Michael BLISS, The Discovery of Insulin, 38, 3: 430-431; Normand PERRON, Un siècle de vie hospitalière au Québec. Les Augustines et l'Hôtel-Dieu de Chicoutimi, 1884-1984, 39, 1: 110-112; S. E. D. SHORTT, Medicine in Canadian Society: Historical Perspectives, 36, 3: 441443

BERNIER, Serge, J. L. GRANATSTEIN, Canada 1957-1967: The Years of Uncertainty and Innovation, 40, 3: 447-450

BLAIN, Jean, Fernand BRAUDEL, dir., Le monde de Jacques Cartier. L'aventure au XVIe siècle, 38, 3: 431-434; Guy FRÉGAULT, Lionel Groulx tel qu' en lui-même: "Guy Frégault à travers Lionel Groulx tel qu'en lui-même» (NC), 36, 4: 569-582

BLISS, Michael, Peter OLIVER, $G$. Howard Ferguson: Ontario Tory, 33, 2: 269-270

BOGLIONI, Pierre, Jean SIMARD, dir., Un patrimoine méprisé. La religion populaire des Québécois, 34, 3: 437-438

BOULAY, Odette et Jacques MATHIEU, Marcel LAFORTUNE, Initiation à la paléographie franco-canadienne. Les écritures des notaires aux XVIIe-XVIIIe siècles, 37, 3: 464-465 et réplique, 38, 2: 275-276

BOULLE, Plerre H., Guide des sources de l' histoire de l'Amérique latine et des Antilles dans les Archives françaises, 39, 4: 606-607
BOURDON, Yves, Alan F. J. ARTIBISE et Paul-André LINTEAU, L'évolution de l'urbanisation au Canada. Une analyse des perspectives et des interprétations, 39, 2 : 272-275

BRADBURY, Bettina, Anita Clair FELLMAN et Veronica STRONG-BOAG, eds., Rethinking Canada: The Promise of Women's History, 40, 3: 454-455; Marie LAVIGNE et Yolande PINARD, Travailleuses et féministes. Les femmes dans la société québécoise, 38, 3: 441-443; Alison PRENTICE et Susan Mann TROFIMENKOFF, eds., The Neglected Majority: Essays in Canadian Women's History, vol. 2, 39, 4: 604-605

BRANDT, Gail Cuthbert, Lucien MICHAUD, Cent ans de vie française à Sudbury, 1883 . 1983, 38, 1: 109-111

BRIĖRE, Jean-Marie, Réginald HAMEL, John HARE et Paul WYCZYNSKI, Dictionnaire pratique des auteurs québécois, 31, 4: 580581

BRUNELLE, Dorval, Michel PRATT, La Grève de la United Aircraft, 34, 2: 285-289; JeanLouis ROY, Le choix d'un pays. Le débat constitutionnel Québec-Canada 1960-1976, 33, 3: 478-480

BRUNET, Michel, Gérald A. BEAUDOIN, Essais sur la Constitution, 34, 3: 440-441; Walter GORDON, A Political Memoir, 33, 1: 83-86; J. L. GRANATSTEIN, The Ottawa Men: The Civil Service Mandarins, 1935-1957, 37, 2: 348-351; Arthur R. M. LOWER, A Pattern for History, 33, 4: 597 598; Joseph SCHULL, Un Grand Patron. Une biographie de Donald Gordon, 35, 4: 599-602; Brian YOUNG, George-Étienne Cartier: Montreal Bourgeois, 36, 2: 278279

BUREAU, Jacques, Alan ARTIBISE, Winnipeg: An Illustrated History, 32, 4: 629-630; Vancouver's First Century: A City Album 1860-1970, 32, 4: 642-643

BURGESS, Joanne, Lucia FERRETTI, Yvan LAMONDE et Daniel LEBLANC, $L a$ culture ouvrière à Montréal (1880-1920). Bilan historiographique, 37, 1: 103-104, réplique, $37,3: 472-473$ et réponse de $\mathrm{J}$. Burgess, 37, 3: 474

BURNS, Robin B., Claudette CARDINAL, The History of Quebec: A Bibliography of Works in English, 37, 3: 455-456; Christopher 
MOORE, The Loyalists: Revolution, Exile, Settlement, 39, 2: 293

CAMPEAU, Lucien, Jean-Pierre ASSELIN, Les Rédemptoristes au Canada. Implantation à Sainte-Anne-de-Beaupré, 1878-1911, 35, 4: 589-590; Marc-André BÉDARD, Les Protestants en Nouvelle-France, 32, 4: 630633; Clarence J. d'ENTREMONT, Histoire du Cap-Sable de l'an mil au traité de Paris, 1763, 36, 3: 431-432; Guy Frégault (19181977). Actes du colloque tenu au Centre de recherche en civilisation canadienne-française de l'Université d'Ottawa, le 7 septembre 1980, recueillis et présentés par Pierre Savard, 36, 4: 606; Gabriel SAGARD, Le grand voyage du pays des Hurons, 31, 1: 113; Bruce G. TRIGGER, The Children of Aataentsic: A History of the Huron People to 1660, 31, 3: 437-440; Marcel TRUDEL, Histoire de la NouvelleFrance, III: La Seigneurie des Cent-Associés 1627-1663, Tome 1: Les Événements, 35, 3: 431-433

CARDIN, Jean-François, Jean-Michel

CATTA, La grève des bûcherons de Rouyn, 1933, 40, 4: 628-629; Louis FOURNIER, F.L.Q. Histoire d' un mouvement clandestin, 37, 2: 343-345

CARELESS, J. M. S., Michael BLISS, A Canadian Millionaire: The Life and Business Times of Sir Joseph Flavelle, 1858-1939, 34, 4: 627-630

CARRIER, Maurice, Madeleine DOYON-FERLAND, Jeux, rythmes et divertissements traditionnels. Textes colligés et présentés par Andrée Paradis, 35, 3: 416-417; Bernard GENEST, Massicotte et son temps, 34, 4: 630-631

CAUCHON, Michel, Robert-Lionel SÉGUIN, dir., Le travail du chaume dans la région du lac St-Pierre, 33, 4: 601-602

CAULIER, Brigitte, Benoît LACROIX et Jean SIMARD, dir., Religion populaire, religion de clercs?, 39, 1: 104-107
CAYA, Marcel, ARCHIVES PUBLIQUES DU CANADA, Documents historiques du gouvernement/Historical Records of the Government of Canada, 34, 2: 275-274; J. Murray BECK, Joseph Howe, vol. I: Conservative Reformer, 1804-1848; vol. II: The Briton Becomes Canadian, 1848-1873, 40, 4: 590-591; Marcel BOULET et Jacques DUCHARME, Répertoire numérique du Fonds de l'Institut agricole d'Oka (E82), 33, 4: 602-603; Terry COOK et Glenn T. WRIGHT, Documents historiques du gouvernement du Canada, 33, 4: 603-604; Yvan LAMONDE, Les bibliothèques de collectivités à Montréal (17e-19e siècle). Sources et problèmes, 34, 4: 652; Robert MIGNER et Robert LÉVESQUE, Les Boss politiques à Montréal. Camillien et les années vingt suivi de Camillien au Goulag. Cartographie du houdisme, 37, 1: 109-110; MINISTERE DES AFFAIRES CULTURELLES, Rapport des Archives nationales du Québec, 1975, 31 , 1: 105-106; J. W. PICKERSGILL, My Years with Louis St. Laurent: A Political Memoir, 31, 1: 106107

CHABOT, Richard, Nicole GAGNON et Jean HAMELIN, dir., L' homme historien, 34, 2: 278-282; Jeanne L'ARCHEVÊQUEDUGUAY, Lettres d' une paysanne à son fils, 31, 4: 583-585

CHAMPAGNE, Édith, Maurice CARRIER et Monique VACHON, Chansons politiques du Québec 1834-1858, 35, 2: 274-275

CHARLAND, Diane, Michel ROBERGE, $\mathrm{La}$ gestion des documents administratifs, 40, 3: 452-453

CHARLAND, Jean-Pierre, Louise B.-LAVOIE, Les débuts du mouvement ouvrier à Sherbrooke, 1873-1919, 34, 3: 457-458

CHOUINARD, Denys, Michael BEHIELS et Ramsay COOK, eds., The Essential Laurendeau, 31, 2: 260-261

CHOUINARD, Yvan, Paul JACOB, Les revenants de la Beauce, 33, 4: 596-597

CLERMONT, Norman, Robert McGHEE, $\mathrm{La}$ préhistoire de l'Arctique canadien, 39, 1: 109-110; James A. TUCK, La préhistoire de Terre-Neuve et du Labrador, 39, 1: 109110 ; L. F. S. UPTON, Micmacs and Colonists: Indian-White Relations in the Maritimes, 1713-1867, 35, 3: 440 


\section{Revue d'histoire de l'Amérique française}

CLICHE, Marie-Aimée, Ghislaine BOUCHER, Dieu et Satan dans la vie de Catherine de Saint-Augustin, (I632-1668), 35, 1: 100101; Jean LECLERC, Le marquis de Denonville, gouverneur de la NouvelleFrance, 1685-1689, 31, 4: 585-586

COHEN, Yolande, Nicole GAGNON et Jean HAMELIN, dir., L'Histoire orale, 34, 2: 277-278

COMEAU, Robert, Jacques COUSINEAU, L'Église d' ici et le social 1940-1960, tome 1: La Commission sacerdotale d'Études sociales, 37, 2: 334-342; Andrée

LÉVESQUE, Virage à gauche interdit. Les communistes, les socialistes et leurs ennemis au Québec 1929 à 1939, 39, 2: 289-292

COOK, Ramsay, Denis MONIÈRE, André Laurendeau et le destin d'un peuple, 38, 4: 604606; Gil RÉMILLARD, Le fédéralisme canadien. Éléments constitutionnels de formation et d'évolution, 35, 3: 426-428

COURVILLE, Serge, Pieter J. DE VRIES et Georgina MacNAB-DE VRIES, They Farmed, Among Other Things..., 39, 2: 281283; Allan GREER, Peasant, Lord, and Merchant: Rural Society in Three Quebec Parishes 1740-1840 (NC), 39, 3: 407-413

COUTURE, Claude, Clinton ARCHIBALD, Un Québec corporatiste?, 38, 3: 427-430; Paul CHAMBERLAND, Un parti pris anthropologique, 38, 3: 434-435

CRUNICAN, Paul, Robert CHOQUETTE, $L$ 'Église catholique dans l'Ontario français du 19e siècle, 40, 4: 592-593

CYR, André, COMMISSION DE LA CAPITALE NATIONALE, Région de la capitale nationale. Bibliographie de l' histoire et du patrimoine/National Capital Region. History and Heritage Bibliography, 31, 1: 88-89

\section{D}

DAIGLE, Jean, Ernest R. FORBES, Maritime Rights. The Maritime Rights Movement, 1919-1927. A Study in Canadian Regionalism, 33, 4: 592-594; Julian GWYN et Christopher MOORE, eds., La Chute de Louisbourg. Le journal du ler siège de
Louisbourg du 25 mars au 17 juillet 1745 par Gilles Lacroix-Girard, 33, 4: 605; JeanWilliam LAPIERRE et Muriel ROY, Les Acadiens, 38, 1: 106-107; Bertha PLOURDE, Mgr L.-J.-Arthur Melanson 1879-1941, 40, 3: 439-441; John G. REID, Acadia, Maine and New Scotland: Marginal Colonies in the Seventeenth Century, 36, 4: 599-600

DAIGLE, Joane, Robert RUMILLY, Boscoville, 34, 3: 458

D'ALLAIRE, Micheline, Dictionnaire biographique du Canada, vol. V: de 1801 à 1820 , 38, 2: 261-262

DAUPHINAIS, Paul, Serge DENIS, Un syndicalisme pur et simple. Mouvements ouvriers et pouvoir publique aux États-Unis, 19191939, 40, 4: 609-610

DE BROU, David, Colin READ et Ronald J. STAGG, eds., The Rebellion of 1837 in Upper Canada , 40, 1: 101-103

DE GROSBOIS, Guy, Claude BERGERON, Index des périodiques d'architecture canadiens, 1940-1980/Canadian Architectural Periodicals Index, 1940-1980, 40, 3: 456

DÉCARIE, Graeme, Gary CALDWELL, Les études ethniques au Québec. Bilan et perspectives, 37, 4: 614-615

DELAGE, Denys, Lucien CAMPEAU, Gannentaha - Première mission iroquoise (16531665), 39, 1: 96-98

DENAULT, Bernard, Danielle NEPVEU, Les représentations religieuses au Québec dans les manuels scolaires de niveau élémentaire 1950-1960, 37, 1: 119-120; Michel THÉRIAULT, Les instituts de vie consacrée au Canada depuis les débuts de la NouvelleFrance jusqu'à aujourd' hui, 36, 4: 609

DESBIENS, Albert, John W. HOLMES, Life with Uncle: the Canadian-American Relationship, 40, 4: 617-618

DÉSCHÊNES, Gaston, Claude LARIVIÈRE, Albert Saint-Martin, militant d'avant-garde (1865-1947), 35, 3: 419-420; QUÉBEC (prov.). Ministère des institutions financières et coopératives. Direction des associations coopératives, Histoire du mouvement coopératif au Québec, 36, 4: 606-607

DÉSILETS, Andrée, ASSEMBLÉE NATIONALE DU QUÉBEC, Débats de l'Assemblée Législative, 8e législature: 1893-1897, 35, 3: 434-435; André BEAULIEU et Jean 
HAMELIN, La Presse québécoise des origines à nos jours, tome 4: 1896-1910, 34, 3: 465; André BEAULIEU et Jean HAMELIN, La presse québécoise des origines à nos jours, tome 5: 1911-1919, 36, 3: 427; André BEAULIEU et Jean HAMELIN, $\mathrm{La}$ presse québécoise, tome VI: 1920-1934, 39, 3: 444; Marc-André BÉDARD, Débats de l'Assemblée législative du Québec: 1899, 39, 3: 443-444; Mgr René BÉLANGER, L'avion à la conquête de la Côte-Nord. Développement de l'aviation sur la CôteNord, 1919-1954, 35, 1: 115-116; Olga B. BISHOP et al., Bibliography of Ontario History, 1967-1976, 35, 3: 435; Brian A. BROWN, The New Confederation: Five Sovereign Provinces, 32, 2: 262-263; Dictionnaire biographique du Canada, vol. XI: 1881-1890, 36, 3: 430-431; Dictionnaire biographique du Canada: Guide de consultation, volumes I à $I V$, de l'an 1000 à 1800 , 36, 3: 430-431; Gary GEDDES, ed., Divided We Stand, 32, 2: 262-263; Michael J. GRIBBON, Walter J. Philips. Un choix de son oeuvre et de ses réflexions, 32, 4: 643644; Ronald D. LAMBERT, The Sociology of Contemporary Quebec Nationalism: An Annotated Bibliography and Review, 39, 3: 445; Jim LOTZ et William METCALFE, Understanding Canada: Regional and Community Development in a New Nation, 32, 2: 266; Jean POIRIER, Regards sur les noms de lieux, 37, 1: 114-116; Virginia R. ROBESON, ed., Debates about Canada's Future, 1868-1896, 32, 2: 272-273; Jocelyn SAINT-PIERRE, Débats de l'Assemblée législative du Québec: 1897-1898, 39, 3: 443-444; Richard SIMEON, ed., Must Canada Fail?, 32, 2: 262-263; George F. G. STANLEY, Les écrits complets de Louis Riel/The Collected Writings of Louis Riel, 40, 3: 429-432; Pierre TRÉPANIER,

Siméon Le Sage. Un haut fonctionnaire québécois face aux défis de son temps (1867. 1909), 36, 4: 601-603; Georges VINCENTHIER, Histoire des idées au Québec. Des troubles de 1837 au référendum de 1980, 38, 4: 611-612

DESJARDINS, Ghislaine, Denise PÉLISSIER, Répertoire numérique détaillé du Fonds de l'Ecole Ménagère provinciale, 35, 3: 438439; Yvonne RIALLAND-MORISSETTE,
Le passé conjugué au présent, 36, 3: 437438

DESLOGES, Yvon, Elinor Kyte SENIOR, British Regulars in Montreal: An Imperial Garrison, 1832-1854, 36, 4: 600-601; Frederick J. THORPE, Remparts lointains. La politique française des travaux publics à TerreNeuve et à l'île Royale, 1695-1758, 35, 3: 430-431

DESROSIERS, Richard, Desmond MORTON et Terry COPP, Working People: An Illustrated History of Canadian Labour, 36, 2: 270-272

DESSUREAULT, Christian, Claude BARIBEAU, La seigneurie de la Petite-Nation 1801-1854. Le rôle économique et social du seigneur, 38, 2: 255-256

DICKASON, Olive Patricia, Donald B. SMITH, Long Lance. The True Story of an Impostor, 37, 1: 116-117

DICKINSON, John A., Yves DURAND, Vivre au pays au XVIIIe siècle. Essai sur la notion de pays dans l'ouest de la France, 40, 2: 289-290; François-Marc GAGNON, Ces hommes dits sauvages. L' histoire fascinante d'un préjugé qui remonte aux premiers découvreurs du Canada et Olive Patricia DICKASON, The Myth of the Savage and the Beginnings of French Colonialism in the Americas: «L'image de l'autre: Européens et Amérindiens» (NC), 39, 2: 263-270; Melvina GALANT, Le sieur de Dièreville. Voyage à l'Acadie, 1699-1700, 40, 1: 113; June HELM, ed., Handbook of North American Indians, vol. 6: Subartic, 36, 4: 603605; Elisabeth JONES, Gentlemen and Jesuits: Quests for Glory and Adventure in the Early Days of New France, 40, 2: 300301; Shepard KRECH III, ed., The Subarctic Fur Trade: Native Social and Economic Adaptations, 39, 3: 420-422; André LACHANCE, La justice criminelle du roi au Canada au XVIIIe siècle. Tribunaux et officiers, 32, 4: 635-636; Yvon LACROIX, Les origines de La Prairie (1667-1697), 36, 1: 110-112; Lawrence M. LANDE, ed., The Rise and Fall of John Law, 1716-1720, 38, 4: 601-602; Edith PILCHER, Castorland: French Refugees in the Western Adirondaks, 1793-1814, 39, 3: 443; Bruce G. TRIGGER, ed., Handbook of North American Indians, vol. 15: Northeast; vol. 6: Subarctic, 36, 4: 603-605 
DIONNE, Bernard, Ian ANGUS, Canadian Bolsheviks: The Early Years of the Communist Party of Canada, 39, 1: 91-93; Terry COPP, Le S.I.T.E. [Syndicat international des travailleurs de l'électricité] au Canada, 35, 3: 415-416; François CYR et Rémi ROY, Éléments d' histoire de la FTQ. La FTQ et la question nationale, 37, 1:97-99; Historiographie du Parti communiste canadien 1960-1982, 37, 2: 309-319

DIONNE, Raoul, Michel ROY, L'Acadie des origines à nos jours. Essai de synthèse historique, 38, 3: 445-447

DORAN-JACQUES, Anne, Hélène CHAPUT, Mère Marie-Du-Rosaire (Henriette Préfontaine 1845-1906 - Huitième supérieure générale 1900-1906), 37, 1: 94-95

DROLET, Gilbert, Jean V. ALLARD et Serge BERNIER, Mémoires du Général Jean V. Allard, 40, 2: 297-298

DUBOIS, Jean-Marie M., Maurice ASSELIN, La colonisation de l'Abitibi, un projet géopolitique, 38, 1: 93-95; Andrée DÉSILETS, Les noms de rues de Sherbrooke (1825. 1980), 38, 2: 258-261; Joseph LALIBERTÉ et Robert LAPLANTE, Agronome-colon en Abitibi, 38, 1: 104-105

DUCHARME, Jacques, ARCHIVES DU CANADA, Communiqué, 35, 1: 99-100 DUCHESNE, Raymond, Denis BARABÉ et Sylvie LALIBERTÉ, dir., Le Jardin botanique de Montréal: 1931 à 1981, 37, 3: 453; Carl BERGER, Science, God, and Nature in Victorian Canada, 38, 4: 591-592; Geoffrey BILSON, A Darkened House: Cholera in Nineteenth-Century Canada, 35, 3: 408411; Sylvio LEBLOND, Médecine et médecins d'autrefois. Pratiques traditionnelles et portraits québécois, 40, 4: 625-626; W. E. Knowles MIDDLETON, Radar Development in Canada: The Radio Branch of the National Research Council of Canada 1939. 1946, 36, 2: 269-270

DUMONT-JOHNSON, Micheline, Francine BARRY, Le travail de la femme au Québec. L'évolution de 1940 à 1970, 31, 2: 255-256; Rosario BILODEAU et Gisèle MORIN, Histoire nationale, 31 , 2: 256-258; Gérard CACHAT, L'Aventure française en Amérique. Un défi 1534-1976, 31, 4: 575; Marguerite JEAN, Évolution des communautés religieuses de femmes au Canada de 1639 à nos jours, 31, 4: 582-583; Germaine
LAPLANTE, Une journaliste intemporelle, Germaine Bernier, 32, 4: 636-637; Élisabeth J. LACELLE, dir., La femme et la religion au Canada français - un fait socioculturel, 35, 3: 436-437; Marie LAVIGNE et Yolande PINARD, Les femmes dans la société québécoise, 32, 1: 101-103

DUPONT, Jean-Claude, Yvan CHOUINARD et Lise CYR, Le Forgeron Émile Asselin, 31, 1: 95-96

DUROCHER, René, Conrad BLACK, Duplessis et Robert Rumilly, Duplessis et son temps: «L'histoire partisane: Maurice Duplessis et son temps vue par Robert Rumilly et Conrad Black» (NC), 31, 3: 407426

$\mathbf{E}$

EID, Nadia F., Léon POULIOT, Monseigneur Bourget et son temps. Tome IV : Affrontement avec l'Institut Canadien, 1858-1870, 31, 1: 107-109; Louis ROUSSEAU, La prédication à Montréal de 1800 à 1830 . Approche religiologique, 34, 1: 115-119

$\mathbf{F}$

FECTEAU, Jean-Marie, Pierre-E. AUDET, Les officiers de justice des origines de la colonie à nos jours, 40, 3: 451-452; André LACHANCE, Crimes et criminels en Nouvelle-France, 39, 1: 102-104; Jacques LAPLANTE, Crime et traitement. Introduction critique à la criminologie, 39, 4: 606; Judy M. TORRANCE, Public Violence in Canada, 1867-1982, 40, 4: 627-628

FLEURENT, Maurice, Noël BAILLARGEON, Le Séminaire de Québec de 1685 à 1760 , 32, 1: $95-98$

FORTIER, Rénald, W. A. B. DOUGLAS, The Official History of the Royal Canadian Air Force, vol. 2: The Creation of a National Air Force, 40, 4: 610-613 
FRENETTE, Yves, Bruno RAMIREZ, Les premiers Italiens de Montréal. L'origine de la Petite Italie du Québec, 39, 3: 432-434; Claude SAVARY, dir., Les rapports culturels entre le Québec et les États-Unis, 40, 1: 109-111

GADOURY, Lorraine, Réal BATES, Les conceptions prénuptiales dans la vallée du Saint-Laurent avant 1725, 40, 2: 301-302

GAFFIELD, Chad, Donald H. AKENSSON, ed., Canadian Papers in Rural History, vol. III, 39, 3: 418-420; Yolande LAVOIE et Christian POUYEZ, Les Saguenayens. Introduction à l' histoire des populations du Saguenay, XVIe-XXe siècles, 39, 3: 422-424

GAGNON, François-Marc, Robert CARON, Un couvent du XIXe siècle. La Maison des Soeurs de la Charité de Québec, 36, 3: 432435; Madeleine GOBEIL-TRUDEAU, Bâtir une église au Québec. Saint-Augustin-deDesmaures: de la chapelle primitive à l'église actuelle, 36, 3: 432-435; Luc NOPPEN, Les églises du Québec (1600-1850), 35, 1: 114-115

GAGNON, Serge, La conversion par l' image. Un aspect de la mission des Jésuites auprès des Indiens du Canada au XVIle siècle, 31, 4: 578-579

GALARNEAU, Claude, Mary ALLODI, Printmaking in Canada: The Earliest Views and Portraits. Les débuts de l'estampe imprimée au Canada. Vues et portraits, 35, 1: 97-98; Marcel LAJEUNESSE, Les Sulpiciens et la vie culturelle à Montréal au XIXe siècle, 37, 4: 618-619

GAUTHIER, Benoît, Guy GAUDREAU, L'exploitation des forêts publiques au Québec, 1842-1905, 40, 4: 626-627

GAUTHIER-LAROUCHE, Georges, JeanClaude DUPONT, dir., Habitation rurale au Québec, 33, 1: 82-83; Raymonde GAUTHIER, Les manoirs du Québec, 31, 2: 265-266

GENEST, Bernard, Jacques DORION, Les écoles de rang au Québec, 33, 4: 589-590
GENEST, Jean-Guy, Bona ARSENAULT, Souvenirs et Confidences, 38, 1: 91-93; Réal BÉLANGER, L'impossible défi. Albert Sévigny et les conservateurs fédéraux (1902-1918), 37, 4: 612-614; Jean GOSSELIN, Portraits de cheminots, 38, 2: 262265; Marcel HUGUET, Réal Caouette. L' homme et le phénomène, 37, 2: 351-352; G.-Raymond LALIBERTÉ, Une société secrète: l'Ordre de Jacques-Cartier, 38, 3: 438-441, réplique, $40,2: 286$ et réponse de J.-G. Genest, 40, 2: 286-288; Jacques PINAULT, Dans les coulisses du pouvoir, 36, 2: 275-277; Bernard SAINT-AUBIN, Duplessis et son époque, 34, 2: 293-295; C. P. STACEY, Canada and the Age of Conflict: A History of Canadian External Policies, vol. 2: 1921-1948 - The Mackenzie King Era, 37, 4: 631-634

GÉRIN-LAJOIE, Jean, Benoît-Beaudry GOURD, Mines et syndicats en AbitibiTémiscamingue 1910-1950, 37, 1: 101-103

GERVAIS, Gaétan, R. Alan DOUGLAS, John Prince 1796-1870. Collection of Documents, 35, 4: 592-594; William F. E. MORLEY, Ontario and the Canadian North, 33, 4: $599-600$

GINGRAS, Yves, Jean-Pierre CHARLAND et Nicole THIVIERGE, Bibliographie de l'enseignement professionnel, 1850-1980 et Jean-Pierre CHARLAND, Histoire de l'enseignement technique et professionnel, 39, 3: 427-430; Madeleine LAVALLÉE, MarieVictorin, un itinéraire exceptionnel: «L'itinéraire du frère Marie-Victorin, É.C. (18851944)» (NC), 39, 1: 77-82

GOUIN, Jacques, Gérard FILTEAU, Le Québec, le Canada et la Guerre 1914-1918, 31, 4: 576-578; Robert RUMILLY, Papineau et son temps, 32, 2: 273-275

GOURD, Benoît-Beaudry, Jean GÉRINLAJOIE, Les métallos 1936 à 1981, 37, 2: 346-348

GRAVEL, Jean-Yves, F. J. HATCH, Le Canada, aérodrome de la démocratie. Le plan d'entraînement aérien du Commonwealth britannique, 1939-45, 38, 1: 103104; S. F. WISE, Canadian Airmen and the First World War: The Official History of the Royal Canadian Air Force, vol. 1, 36, 3: 445-447 
GRIMARD, Jacques, Nelson FECTEAU et Jean-Claude POULIN, La Cité de l'or blanc. Thetford Mines, 1876-1976, 31, 2: 271-273; Gérard FILTEAU, La naissance d'une Nation. Tableau de la NouvelleFrance en 1755, 32, 2: 259-261; Normand PAQUIN, Histoire de l'Abitibi-Témiscamingue, 34, 2: 284-285

\section{H}

HAMEL, Thérèse, Micheline DUMONT et Nadia Fahmy EID, Maîtresses de maison, maîtresses d'école. Femmes, famille et éducation dans l'histoire du Québec, 38, 4: 594-596

HARDY, René, Fabien LAROCHELLE, Shawinigan depuis 75 ans - (1900-1975), 31, 2: 266-268; Bx de MAZENOD, Lettres aux correspondants d'Amérique, 1841-1850, 32, 1: 103-104; J.-Alide PELLERIN, Yamachiche et son histoire, 1672-1978, 36, 4: -598-599

HEAP, Ruby, Dominique CLIFT et Sheila McLEOD-ARNOPOULOS, Le fait anglais au Québec, 34, 1: 101-105; Bernard LEFEBVRE, L'école sous la mitre, 35, 3: 421-424

HENLEY, Kevin, Ben FORSTER, A Conjunction of Interests: Business, Politics, and Tariffs 1825-1879, 40, 4: 595-596

\section{I}

IGARTUA, José E., Robert ARMSTRONG, Structure and Change: An Economic History of Quebec: «Le Québec de Pangloss» (NC), 39, 2: 253-261, réplique, 40,2: 273 277 et réponse de J. Igartua, 40, 2: 277-283; Dictionnaire biographique du Canada, vol. IV: de 1771 à 1800, 36, 4: 586-587; Dale MIQUELON, Dugard of Rouen: French Trade to Canada and the West Indies, 1729$1770,33,3: 468-471$

\section{$\mathbf{J}$}

JAENEN, Cornelius J., Bernard ARCAND et Sylvie VINCENT, L'Image de l'Amérindien dans les manuels scolaires du Québec, 34, 3: 460-462; Donald B. SMITH, Le «Sauvage» pendant la période héroïque de la Nouvelle-France (1534-1633) d'après les historiens canadiens-français des XIXe et XXe siècles, 34, 3: 459-460

JANSON, Gilles, Carol COUTURE et JeanYves ROUSSEAU, Les archives au XXe siècle. Une réponse aux besoins de l'administration et de la recherche, 37, 3: 456-459

JOHNSON, Micheline, voir DUMONTJOHNSON, Micheline

JONES, Richard, I. M. ABELLA, D.J. BERCUSON, R.C. BROWN, J.L. GRANATSTEIN et H.B. NEATBY, Twentieth Century Canada, 38, 4: 606-610; Paul AUBIN, Bibliographie de l' histoire du Québec et du Canada, 1976-1980, tome I et II, 39, 3: 439-440; Richard Dalton BASHAM, Crisis in Blanc and White: Urbanization and Ethnic Identity in French Canada, 34, 3: 438440; Gérald-A. BEAUDOIN, Le partage des pouvoirs, 35, 4: 590-592; Gilles BOURQUE et Anne LÉGARÉ, Le Québec: la question nationale, 36, 2: 266-269; Dorval BRUNELLE, La désillusion tranquille, 33, 1: 79-81; Norman BUCHIGNANI, Doreen M. INDRA et Ram SRIVASTIVA, Continuous Journey: A Social History of South Asians in Canada, 39, 4: 602-604; Mario CARDINAL, Vincent LEMIEUX et Florian SAUVAGEAU, Si l'Union nationale m'était contée..., 32, 2: 257-258; Louis-Marie COTÉ, Bibliographie de l'histoire du Québec et du Canada, 1976-1980, tome I et II, 39, 3: 439-440; Michael S. CROSS et Gregory S. KEALEY, eds., Readings in Canadian Social History, vol. 5: Modern Canada: 1930-1980's, 38, 4: 606610; Dictionnaire biographique du Canada, volume VIII, de 1851 à 1860, 40, 3: 453; J. L. GRANATSTEIN et J.M. HITSMAN, Broken Promises: A History of Conscription in Canada, 31, 3: 432-434; Paul GROS D'AILLON, Daniel Johnson, l'égalité avant l' indépendance, 34, 2: 282-284; John W. HOLMES, The Shaping of Peace: 
Canada and the Search for World Order, 1943-1957, vol. II , 39, 3: 436-438; INSTITUT QUÉBÉCOIS DE RECHERCHE SUR LA CULTURE, Statistiques culturelles $d u$ Québec, 1971-1982, 40, 2: 303-304; Louis LAROCHELLE, En flagrant délit de pouvoir. Chronique des événements politiques de Maurice Duplessis à René Lévesque, 37, 1: 104-105; Maurice LEBEL, Association canadienne d'éducation de langue française, 35e anniversaire, 1947-1982. Souvenirs historiques, 39, 3: 444-445; Paul-André LINTEAU, René DUROCHER et JeanClaude ROBERT, Histoire du Québec contemporain, vol. 1: De la Confédération à la crise, 34, 4: 642-644; Christina McCALL-NEWMAN, Les Rouges. Un portrait intime du parti libéral, 38, 3: 444-445; Kenneth McROBERTS et Dale POSGATE, Quebec: Social Change and Political Crisis, 35, 3: 424-426; Henry MILNER, Politics in the New Quebec, 33, 1: 95-97; Desmond MORTON, A Short History of Canada, 38, 4: 606-610; André PATRY, Le Québec dans le monde, 35, 2: 284-286; Alain PON-

TAUT, René Lévesque ou "l' idéalisme pratique», 38, 2: 268-270; Ronald RUDIN, The Forgotten Quebecers: a History of EnglishSpeaking Quebec, 1759-1980, 40, 1: 97-98; Bernard SAINT-AUBIN, King et son époque, 37, 2: 356-358; Michel SARRABOURNET, L'Affaire Roncarelli: Duplessis contre les Témoins de Jéhovah, 40, 3: 444445; Allen SEAGER et John Herd THOMPSON, Canada 1922-1939: Decades of Discord, 39, 4: 600-602; Carl F. SMITH, French-English Relations in Canada, 35, 3: 439; Susan Mann TROFIMENKOFF, The Dream of Nation: A Social and Intellectual History of Quebec, 37, 4: 624-626; Bernard L. VIGOD, Quebec Before Duplessis: The Political Career of Louis-Alexandre Taschereau, 40, 3: 441-443

\section{K}

KEYES, John, Graeme WYNN, Timber Colony: A Historical Geography of Early
Auteurs de comptes rendus

Nineteenth Century New Brunswick, 36, 1: 126-128

KEYSERLINGK, Robert H., J. L. GRANATSTEIN, A Man of Influence: Norman A. Robertson and Canadian Statecraft, 191968, 39, 3: 434-436

KLEIN, Juan-Luis, Nicole BERTHIAUME, Rouyn-Noranda. Le développement d'une agglomération minière au coeur de l' Abitibi-Témiscamingue, 37, 3: 453-455

KUCZEWSKI, André C., Merrily WEISBORD, The Strangest Dream: Canadian Communists, the Spy Trials, and the Cold War, 38, 2: 271-275

$\mathbf{L}$

LACHANCE, André, John Alexander DICKINSON, Justice et justiciables. La procédure civile à la Prévôté de Québec, 1667-1759, 37, 3: 459-461

LACHANCE, Paul, Marc de VILLIERS DU TERRAGE, The Last Years of French Louisiana, 39, 1: 117-120

LACROIX, Benoît, Maurice CARRIER et Monique VACHON, Chansons politiques du Québec 1765 à 1883, 32, 1: 98-100; Micheline D'ALLAIRE, Vingt ans de crise chez les religieuses du Québec 1960-1980, 38, 2: 256-258; Jean-Claude DUPONT, Héritage d'Acadie, 31, 2: 263-265; Nicole GUILBAULT, Henri Julien et la tradition orale, 35, 3: 419; Gabriel LABBÉ, Les Pionniers du disque folklorique québécois I920 à 1950, 32, 1: 100; Micheline LACHANCE, Le Frère André: «Hagiographie et historiographie. En marge d'un livre de Micheline Lachance sur le Frère André» (NC), 35, 2: 263-267; Conrad LAFORTE, Le catalogue de la chanson folklorique française. I: Chansons en laisse, 31, 3: 436437; Carmen ROY, Littérature orale en Gaspésie, 36, 4: 608-609

LAFORCE, Hélène, Charlotte FÜHRER, The Mysteries of Montreal. Memoirs of a Midwife, 39, 3: 425-427 
LAFRANCE, Marc, Réal BRISSON, La charpenterie navale à Québec sous le Régime français, 38, 1: 99-100

LAHAISE, Robert, Armand CARDINAL, Histoire de Saint-Hilaire, 35, 1: 116-117; Nathalie CLERK, Le style palladien dans l'architecture au Canada, 39, 2: 275-277; COMMUNAUTÉ URBAINE DE MONTRÉAL, Les édifices scolaires, 35, 3: 435436; COMMUNAUTÉ URBAINE DE MONTRÉAL, Les édifices publics, 36, 3 : 448; Jean-Claude DUPONT et Jacques MATHIEU, dir., Héritage de la francophonie canadienne - Traditions orales, 40, 4: 624-625; Yvan FORTIER, Menuisier charpentier. Un artisan du bois à l'ère industrielle, 35, 3: 417-418; Georges GAUTHIER-LAROUCHE, Évolution de la maison rurale traditionnelle dans la région de Québec (étude ethnographique), 31, 1: $97-$ 98; Leslie MAITLAND, L'architecture néoclassique au Canada, 39, 2: 275-277; Raymond MONTPETIT, Le temps des Fêtes au Québec, 33, 3: 471-473; SOCIÉTÉ HISTORIQUE DE MONTRÉAL, Montréal, artisans, histoire, patrimoine, 34, 3: 465-466; Janet WRIGHT, L'architecture pittoresque au Canada, 39, 2: 275-277

LAJEUNESSE, Marcel, Stanley Brice FROST, McGill University: For the Advancement of Learning, vol. 1: 1801-1895, 35, 2: 275278; Claude GALARNEAU, Les collèges classiques au Canada français (1620-1970), 34, 3: 449-451; Jean LAFLAMME et Rémi TOURANGEAU, L'Église et le théâtre au Québec, 35, 1: 108-111; Jean-Paul de LAGRAVE, Fleury Mesplet (1734-1794): diffuseur des Lumières au Québec, 40, 1: 98-100

LALANCETTE, Mario, B. A. BALCOM, La pêche de la morue à l'lle Royale, 17131758, 39, 4: 593-595; Jacques BLOUIN et Philippe DUBÉ, Deux cents ans de villégiature dans Charlevoix. L' histoire du pays visité, 40, 4: 588-590; Gaston DESCHÊNES, Portraits de Saint-Jean-PortJoli, 38, 3: 450; Daniel FRANCIS, Arctic Chase: A History of Whaling in Canada's North, 38, 4: 596-597; Bruce W. FRY, «Un air de fort" - Les fortifications de Louisbourg, 39, 4: 593-595; Roch SAMSON, Pêcheurs et marchands de la baie de Gaspé au XIXe siècle, 39, 4: 595-597; Peter J. A.
WADDELL et Walter ZACHARCHUK, Le recouvrement du Machault. Une frégate française du XVIIIe siècle, 39, 4: 593-595

LALONDE, André-N., Diane PAYMENT, Batoche (1870-1910), 38, 2: 267-268; A. I. SILVER, The French-Canadian Idea of Confederation 1864-1900, 36, 3: 443-445

LAMARRE, Jean, Jacques ROUILLARD, $A h$ les États! Les travailleurs canadiens-français dans l'industrie textile de la NouvelleAngleterre d'après le témoignage des derniers migrants, 39, 4: 599-600

LAMBERT, James H., Dom Guy-Marie OURY, Mgr Briand, évêque de Québec et les problèmes de son époque, 39, 4: 608609; SOCIÉTÉ HISTORIQUE ACADIENNE, Les Cahiers, 35, 4: 602-603

LAMONDE, Yvan, Jacques BELLEAU, René HARDY et Guy TRÉPANIER, La Mauricie et les Bois-Francs. Inventaire bibliographique 1760-1975, 31 , 3: 434-436; Maurice LEMIRE, dir., Dictionnaire des oeuvres littéraires du Québec, tome 1: des origines à 1900, 31, 4: 586-589; Claude LESSARD, Le Séminaire de Nicolet 1803 à 1969, 35, 1 : 111-113; Yves LEVER et Pierre PAGEAU, Cinémas canadien et québécois. Notes historiques, 31, 2: 269-271; Christiane TREMBLAY-DAVIAULT, Un cinéma orphelin. Structures mentales et sociales du cinéma québécois (1942-1953), 38, 3: 447-449; Milada VLACH et Yolande BUONO, Catalogue de la Bibliothèque nationale du Québec. Laurentiana parus avant 1821, 31, 1: 111-113

LANDRY, Yves, Honorius PROVOST, Les premiers Anglo-Canadiens à Québec. Essai de recensement 1759-1775, 38, 2: 270-271

LANTHIER, Pierre, André BOLDUC, Clarence HOGUE et Daniel LAROUCHE, Québec, un siècle d'électricité, 34, 3: 454-457; Roger LACASSE, Baie James, une épopée. $L$ 'extraordinaire aventure des derniers des pionniers, 38, 3: 436-437; Jorge NIOSI, La bourgeoisie canadienne. La formation et le développement d' une classe dominante, 36 , 2: $273-275$

LAPERRIÈRE, Guy, Richard ARÈS, Le Père Joseph-Papin Archambault, s.j. (18801966). Sa vie, ses oeuvres, 37, 4: 611-612; Philippe ARIÈS, L'homme devant la mort et Paul JACOB, Les revenants de la Beauce: «Les attitudes devant la mort: sur deux 
ouvrages récents» (NC), 32, 2: 251-255; Pierre BRAULT, L'Acadie et son église, 33, 4: 604-605; Pierre-B. CADIEUX ET Réal FORTIN, Les constructions militaires du Haut-Richelieu (guide touristique), 33, 4: 604-605; Pierre-B. CADIEUX, Croisière sur le Richelieu historique, 33, 4: 604-605; Lionel FORTIN, Le maire Nelson Mott et l'histoire de Saint-Jean, 33, 4: 604-605; Réal FORTIN, Bateaux et épaves du Richelieu, 33, 4: 604-605; Réal FORTIN, Petite histoire de Saint-Luc, 33, 4: 604-605; René HARDY, Les zouaves. Une stratégie du clergé québécois au XIXe siècle, 35, 1: 106108; Jean HOUPERT, Les Lorrains en Amérique du Nord, 39, 4: 607-608; Richard JONES, L'idéologie de "L'Action catholique» (1917-1939), 32, 2: 263-265; Ambroise LAFORTUNE, Par les chemins d'Ambroise, 38, 3: 437-438; Jacques LANGLAIS, Les jésuites du Québec en Chine, 1918-1955, 34, 4: 635-636; Paul-François SYLVESTRE, Les communautés religieuses en Ontario français. Sur les traces de Joseph Le Caron, 40, 2: 303; Roch TANGUAY et Jean-Yves THÉBERGE, ...à pied dans le vieux Saint-Jean, 33, 4: 604-605; Paul-André TURCOTTE, Les chemins de la différence. Pluralisme et aggiornamento dans l'après-concile, 39, 2: 298-301; PaulAndré TURCOTTE, L'éclatement d'un monde. Les Clercs de Saint-Viateur et la Révolution tranquille, 39, 2: 298-301; Nive VOISINE, Louis-François Laflèche, deuxième évêque de Trois-Rivières, tome 1: Dans le sillage de Pie IX et de Mgr Bourget (1818-1878), 34, 3: 463-464

LAPOINTE, Marie, John HEMMING, Red Gold: The Conquest of the Brazilian Indians, 33, 1: 87-88

LAROCQUE, Paul, Jules BÉLANGER, Pierre DANSEREAU, Marc DESJARDINS et Yves FRENETTE, Histoire de la Gaspésie, 36, 2: 264-265

LaROSE, André, Recensement de la ville de Québec en 1818 par le curé Joseph Signaÿ (présentation par l'abbé H. Provost), 31, 1: 109-111

LAURENCE, Gérard, Paul RUTHERFORD, The Making of the Canadian Media, 34, 2: 289-293

LAURENDEAU, Yves, Denis MONIÈRE, André Laurendeau et le destin d' un peuple:
«En guise de supplément au Laurendeau de Monières;» (NC), 38, 1: 73-89

LAURIN, Serge, Mario FILION, Une histoire des Pays-d'en-Haut, 36, 3: 449

LAVALLÉE, André, Léon POULIOT, Monseigneur Bourget et son temps - tome V - Les derniers combats: 1 . Le démembrement de la paroisse Notre-Dame 1865; 2. Vingt-cinq années de luttes universitaires 1851-1876, 31, 2: 273-275

LAVALLÉE, Jean-Guy, Noël BAILLARGEON, Le Séminaire de Québec de 1760 à 1800, 37, 2: 321-323; Ramsay COOK, The Regenerators: Social Criticism in Late Victorian English Canada, 40, 1: 105-106;

Paul-André LABERGE, L'Université Laval (1952-1977). Vers l'autonomie, 35, 4: 597; Jean PROVENCHER, C'était l'automne. La vie rurale traditionnelle dans la vallée du Saint-Laurent, 39, 1: 112-113; Jean PROVENCHER, C'était l'été. La vie rurale traditionnelle dans la vallée du Saint-Laurent; Jean PROVENCHER et Johanne BLANCHET, C'était le printemps, 38, 2: 279-280; Jean-Claude DUBÉ, Les intendants de la Nouvelle-France, 39, 3: 415-417 LAVILLE, Christian, Michel ALLARD et al., Histoire nationale du Québec, de sa découverte à aujourd' hui, 35, 3: 407-408; Gaston DESCHÊNES, Ensemble! Revue de la coopération (1940-1951), 31, 3: 427-429 (repris, 32, 4: 633-635); Paul LAROCQUE, Pêche et coopération au Québec, 34, 4: 636-639; Karl PELLENS, Siegfried QUANDT et Hans SÜSSMUTH, dir., Geschichtskultur - Geschichtsdidektik: Internationale Bibliographie, 39, 3: 439

LAVOIE, Elzéar, Jean-Pierre KESTEMAN, «Le Progrès» (1874-1878) / Étude d' un journal de Sherbrooke, 34, 4: 631-635; Paul RUTHERFORD, A Victorian Authority: The Daily Press in Late Nineteenth Century Canada, 39, 1: 113-117

LAVOIE, Yolande, Iain R. MUNRO, Canada: Origins and Options - Immigration, 33, 4: 600-601

LE BLANC, Alonzo, Jacques COTNAM, Le théâtre québécois, instrument de contestation sociale et politique, 31, 1: 92-95

LEDUC, Marcel, Jean-Pierre CHARLAND et Marîse THIVIERGE, Bibliographie de l'enseignement professionnel 1850-1980, 36, 3: 447 
LEGAULT, Roch, George F. G. STANLEY, The War of 1812: Land Operations, 39, 3 : 417-418

LEMIEUX, Lucien, Gilles CHAUSSÉ, JeanJacques Lartigue premier évêque de Montréal, 35, 3: 413-414

LEMIEUX, Vincent, Dale POSTGATE et Kenneth McROBERTS, Développement et modernisation du Québec, 37, 4: 626-628

LESPÉRANCE, André, Robert BOURBEAU et Jacques LÉGARÉ, Évolution de la mortalité au Canada et au Québec 1831-1931, 37, 2: 324-325

LÉTOURNEAU, Jocelyn, K. J. REA, The Prosperous Years: The Economic History of Ontario, 1939-75, 40, 4: 618-622

LÉVESQUE, Andrée, Marcel FOURNIER, Communisme et anticommunisme au Québec (1920-1950), 34, 3: 441-444; Hélène LAFORCE, Histoire de la sage-femme dans la région de Québec, 39, 3: 424-425

LEVITT, Joseph, Robert BOTHWELL, Ian DRUMMOND et John ENGLISH, Canada since 1945: Power, Politics, and Provincialism, 36, 3: 427-429; David ROME, Clouds in the Thirties: On Antisemitism in Canada, 33, 3: 476-478

LIMOGES, Camille, Richard A. JARRELL et Arnold E. ROOS, eds., Critical Issues in the History of Canadian Science, Technology and Medecine/Problèmes cruciaux de l'histoire de la science, de la technologie et de la médecine au Canada, 40, 2: 294-297

LINTEAU, Paul-André, Alan F. J. ARTIBISE et Gilbert A. STELTER, Canada's Urban Past: A Bibliography to 1980 and Guide to Canadian Urban Studies, 36, 4: 583-584

LITALIEN, Raymonde, Étienne TAILLEMITE, Bougainville et ses compagnons autour du monde, 1766-1769, 32, 2: 275-277

LITTLE, J. I., Marcel BELLAVANCE, Un village en mutation: Compton, Québec, 1880 1920, 37, 1: 92-94, réplique, 37, 3: 474-477 et réponse de J.I. Little, 37, 3: 477-479; Gilles PARENT, Deux efforts de colonisation française dans les Cantons de l'Est, 1848 et 1851, 34, 4: 647-650; Normand SÉGUIN, dir., Agriculture et colonisation au Québec. Aspects historiques, 37, 2: 358360

LONCOL, Jean-Marie, Claude Bonaparte AUGUSTE et Marcel Bonaparte
AUGUSTE, Les déportés de SaintDomingue. Contribution à l' histoire de l' $E x$ pédition française de Saint-Domingue (1802-1803), 36, 1: 107-109 Gabriel DEBIEN, Guillaume Mauviel Évêque constitutionnel de Saint-Domingue (18011805), 37, 1: 99-101

M

MAILHOT, Laurent, Maurice LEMIRE, dir., Dictionnaire des oeuvres littéraires du Québec, vol. 1: Des origines à 1900; vol. 2: 1900-1939; vol. 3: 1940-1959, 36, 4: 587589; Lucie ROBERT, Discours critique et discours historiques dans le Manuel d' histoire de la littérature canadienne de langue française de Mgr Camille Roy, 36, 3: 438439

MANN TROFIMENKOFF, Susan, voir TROFIMENKOFF, Susan Mann

MARCIL-LACOSTE, Louise, Yvan LAMONDE, La philosophie et son enseignement au Québec (1665-1920), 35, 2: 278-281

MARSAN, Jean-Claude, Luc NOPPEN, Claude PAULETTE et Michel TREMBLAY, Québec, trois siècles d' architecture, 34, 4: 645647

MASSÉ, Georges, Daniel LATOUCHE, À la remorque des transports, 37, 1: 105-109

MATHIEU, Jacques, Lionel ALLARD, L'Ancienne-Lorette, 34, 4: 650-651; Jean BOURASSA, Index des volumes I à XVII (19601976) Recherches sociographiques, 31, 4 : 591-592; Gabriel DEBIEN, Les esclaves aux Antilles françaises (XVIle et XVIIIe siècles), 31, 2: 261-262; Charles H. FOSS et Richard H. VROOM, Cabinetmakers of the Eastern Seabord: A Study of Early Canadian Furniture, 32, 4: 643; A: J. B. JOHNSTON, L'été de 1744: la vie quotidienne à Louisbourg au XVIIIe siècle, 37, 4: 616-618; Hélène LAFORTUNE et Normand ROBERT, Inventaire des minutes notariales de Jacques-Eugène Faribault, 1831-1840, 35, 4: 598-599; Marcel LAFORTUNE, Initiation à la paléographie franco-cana- 
dienne. Les écritures des notaires aux XVIIe-XVIIIe siècles, 37, 3: 464-465 et réplique, 38, 2: 275-276; Mélanges d'histoire du Canada français offerts au professeur Marcel Trudel, 32, 4: 637-639; JeanJacques MESSIER, Bibliographie relative à la Nouvelle-France, 33, 3: 467-468; RobertLouis STEIN, The French Slave Trade in the Eighteenth Century: An Old Regime Business, 35, 2: 289-290; Paul TERRIEN, Québec à l'âge de la voile, 39, 4: 608

McCALLA, Douglas, Ronald RUDIN, Banking en français: The French Banks of Quebec, 1835-1925, 40, 4: 596-598

MICHEL, Louis, Corinne BEUTLER, Olivier BUCHSENSCHUTZ, Marceau GAST et François SIGAUT, dir., Les techniques de conservation des grains à long terme. Leur rôle dans la dynamique des systèmes de cultures et des sociétés, 40, 2: 299-300

MONET, Jacques, R. H. HUBBARD, Rideau Hall - An Illustrated History of Government House, Ottawa, from Victorian Times to the Present Day, 33, 1: 89-90 David B.

KNIGHT, A Capital for Canada: Conflict and Compromise in the 19th Century, 33, 1: 90-92; David B. KNIGHT, Choosing Canada's Capital: Jealousy and Fiction in the 19th Century, 33, 1: 90-92;

MONIÈRE, Denis, Jean-Paul BERNARD, Les rébellions de 1837-1838 - Les patriotes du Bas-Canada dans la mémoire collective et chez les historiens, 38, 1: 97-98

MONTMINY, Jean-Paul, Richard ALLEN, The Social Passion: Religion and Social Reform in Canada, 1914-1928, 31, 1: 83-85

MONTPETIT, Raymond, Roger LEVASSEUR, Loisir et Culture au Québec, 38, 1: 108-109

MORIN, Claude, Pierre CHAUNU, Histoire, science sociale. La durée, l'espace et l'homme à l'époque moderne, 31, 1: 89-92

MORTON, Desmond, Jacques GOUIN et al., Bon coeur et bon bras. Histoire du régiment de Maisonneuve, 1880-1980, 36, 4: 591593; 37, 1: 120

MOUSSETTE, Marcel, Louise TROTTIER, Les Forges - Historiographie des Forges du Saint-Maurice, 35, 4: 603-604
$\mathbf{N}$

NEATBY, H. Blair, James STRUTHERS, No Fault of Their Own: Unemployment and the Canadian Welfare State 1914-1941, 37, 4: 634-635

NELLES, H. V., William M. BAKER, Timothy Warren Anglin, 1822-96: Irish Catholic Canadian, 35, 2: 269-270

NIOSI, Jorge, Peter C. NEWMAN, Les nouveaux riches - L'establishment canadien II, 37, 2: 354-356

OUELLET, Fernand, Elaine Allan MITCHELL, Fort Timiscaming and the Fur Trade, 33, 1: 97-98; Gérard PARIZEAU, La vie studieuse et obstinée de Denis-Benjamin Viger, 36, 4: 597-598

OWRAM, Doug, Joseph LEVITT, A Vision Beyond Reach - A Century of Images of Canadian Destiny, 37, 4: 621-623

$\mathbf{P}$

PANNETON, Jean, Julien DUHAIME et Rémi TOURANGEAU, dir., 125 ans de théâtre au Séminaire de Trois-Rivières, 39, 4: 609610

PARISEAU, Jean, W. A. B. DOUGLAS et Brereton GREENHOUS, Out of the Shadows: Canada in the Second World War, 31, 3: 429-432; Grant MacEWAN, French in the West/Les Franco-Canadiens dans l'Ouest, 39, 3: 430-432; James G. MacGREGOR, A History of Alberta, 31, 1: 103105; Desmond MORTON, Canada and War: A Military and Political History, 36, 4: 595-597; Desmond MORTON, A Military History of Canada, 40, 2: 293-294 
PARMENTIER, Francis, Yvan LAMONDE, dir., L'imprimé au Québec. Aspects historiques (18e-20e siècle), 38, 4: 598-601; Sophie-Laurence LAMONTAGNE, L' hiver dans la culture québécoise (XVIIe-XIXe siècles), 37, 4: 619-621

PAYMENT, Diane Paulette, Bernard SAINTAUBIN, Louis Riel - Un destin tragique, 39, 4: $597-598$

PELLETIER, Réjean, Pierre DROUILLY, Statistiques électorales fédérales du Québec, 1867-1980, 38, 3: 450-451

PERIN, Roberto, Tina IOANNOU, La communauté grecque du Québec, 38, 3: 435-436

PLAMONDON, Lilianne, Hélène PELLETIERBAILLARGEON, Marie Gérin-Lajoie, 40, 4: 601-602; Ruth Roach PIERSON, "They're Still Women After All»: The Second World War and Canadian Womanhood, 40, 3: 445-447

POIRIER, Claude, Philippe BARBAUD, Le choc des patois en Nouvelle-France. Essai sur l' histoire de la francisation au Canada, 39, 1: 93-95

POIRIER, Marie, Barry BROADFOOT, The Immigrant Years: From Britain and Europe to Canada, 1945-1967, 40, 4: 615-616

POZZO, Jeannine, Alan ARTIBISE, Winnipeg: An Illustrated History, 32, 4: 629-630; Vancouver's First Century: A City Album 18601970, 32, 4: 642-643

PRÉVOS, André, Gérard J. BRAULT, The French-Canadian Heritage in New England, 40, 1: 103-105; Glenn CONRAD, ed., The Cajuns: Essays on Their History and Culture (NC), 34, 1: 95-98; Liliane CRÉTÉ, La vie quotidienne en Louisiane: 1815 1830, 32, 2: 259; Steven DEL SESTO et Jon L. GIBSON, eds., The Culture of Acadiana: Tradition and Change in South Louisiana (NC), 34, 1: 95-98; John Robert McNEILL, Atlantic Empires of France and Spain: Louisbourg and Havana 1700-1763, 40, 4: 586-588; Johnny MONTBARBUT, Les colons de l'Aunis et de la Saintonge au Canada. Régime français, 1608-1763, 39, 3: 441-442; James Hill PARKER, Ethnic Identity: The Case of the French Americans, 37, 4: 628-629; Louise PÉLOQUIN-FARE, L'identité culturelle. Les Franco-Américains de la Nouvelle-Angleterre, 38, 4: 610-611; William Faulkner RUSHTON, The Cajuns: From Acadia to Louisiana, 33, 1: 98-100
PRÉVOST, Jean, Marthe FARIBAULT-BEAUREGARD, La population des forts français d'Amérique (18e siècle), 37, 2: 342-343

PROVOST, Honorius, Guy GIGUÈRE, Luc NOPPEN et Jean RICHARD, La Maison Maizerets - Le château Bellevue, 32, 4: 639640

$\mathbf{R}$

RAMIREZ, Bruno, Brian D. PALMER, Working-Class Experience: The Rise and Reconstitution of Canadian Labour, 1800 1980, 39, 2: 296-298

REA, J. E., Desmond MORTON, A Peculiar Kind of Politics: Canada's Overseas Ministry in the First World War, 37, 3: 466-467

ROBERT, Jean-Claude, Alain CLAVET, Guide to Canadian Photographic Archives/ Guide des archives photographiques canadiennes, 34, 1: 101; Jean HAMELIN et Jean PROVENCHER, Brève histoire du Québec, 37, 4: 615-616; Robert SWEENY, A Guide to the History and Records of Selected Montreal Business Before 1947/Guide pour l'étude d'entreprises montréalaises et leurs archives avant 1947, 34, 1: 119-120

ROBITAILLE, Jacques, Paul-André LINTEAU et Jean-Claude ROBERT, Le Montréal préindustriel (1760-c1850), vol.39 de la série Histoire du Canada en images, 36, 3: 449450

ROBY, Yves, Marie-Louise BONIER, Début de la colonie franco-américaine de Woonsocket, Rhode Island, 36, 2: 265-266; Jacques LAMARCHE, Cyrille Vaillancourt, homme d'action, homme d' unité, coopérateur émérite (1892-1969), 34, 4: 651-652; Robert B. PERREAULT, Elphège-J. Daignault et le mouvement sentinelliste-Manchester, New Hampshire, 36, 1: 119-120; Robert B. PERREAULT, La presse franco-américaine et la politique. L'oeuvre de Charles-Roger Daoust, 36, 1: 118-119

ROUILLARD, Jacques, David J. BERCUSON, Fools and Wise Men: The Rise and Fall of the One Big Union, 33, 4: 587-589; Guy BOISCLAIR, Jean-Pierre KESTEMAN et 
Jean-Marc KIROUAC, Histoire du syndicalisme agricole au Québec, UCC-UPA, 1924-1984, 39, 1: 99-102; Fernand DUMONT, Jean HAMELIN et Jean-Paul MONTMINY, dir., Idéologies au Canada français, 1940-1976, 37, 3: 461-463; Eugene FORSEY, Trade Unions in Canada 1812-1902, 37, 2: 343; Robert GERMAIN, Le mouvement infirmier au Québec. Cinquante ans d' histoire, 40, 4: 622-623; Fernand HARVEY, Le mouvement ouvrier au Québec, 34, 3: 452-453; Fernand HARVEY, Révolution industrielle et travailleurs. Une enquête sur les rapports entre le capital et le travail au Québec à la fin du 19e siècle, 33, 2: 267-268; Guy BOISCLAIR, Normand LAFLEUR, Les «Chinois» de l'Est ou la vie quotidienne des Québécois émigrés aux États-Unis de 1840 à nos jours, 35, 4: 597-598

ROUSSEAU, Louis, Nicole GAGNON et Jean HAMELIN, Histoire du catholicisme québécois, vol. III: Le XXe siècle, tome 1: 1898 1940 et Jean HAMELIN, Histoire du catholicisme québécois, vol. III, Le XXe siècle, tome 2: De 1940 à nos jours (NC), 39, 1: 83-89; Pierre SAVARD, Aspects du catholicisme canadien-français au XIXe siècle, 35, 2: 286-289

ROY, Fernande, Daniel LATOUCHE et Diane POLIQUIN-BOURASSA, Le Manuel de la parole. Manifeste québécois, 34, 4: 639-642

ROY, Jean, Les Cahiers nicolétains, vol. 1 , no I (février 1979), 33, 2: 280; Jacques CROCHETIÈRE, Danièle DOUCET et Josée DUPONT, Jadis une forêt, une rivière. La vie quotidienne à Manseau de 1890 à 1980 , 35, 4: 604; Rodolphe FOURNIER, Lieux et monuments historiques de Trois-Rivières et environs, 34, 2: 276-277; Raymonde GAUTHIER, Trois-Rivières disparue, ou presque, 35, 1: 117

RUDDEL, D. T., M.-A. BLUTEAU, J.-P. CHARLAND et N. THIVIERGE, Les cordonniers, artisans du cuir, 38, 2: 277-279; Jean-Claude DUPONT et Jacques MATHIEU, dir., Les métiers du cuir, 38, 2: 277-279

RUDIN, Ronald, Judith NEFSKY, Paule OBERMEIR et David ROME, Les Juifs au Québec. Bibliographie rétrospective annotée, 36, 4: 608
RYERSON, Stanley Bréhaut, Jacques ROUILLARD, Les syndicats nationaux de 1900 à 1930: «A propos de Les syndicats nationaux... de Jacques Rouillard» (NC), 35, 3: $397-406$

$\mathbf{O}$

SAINT-AMANT, Jean-Claude, Pierre VALLIÈRES, Les scorpions associés, 33, 2: 278-279

SAVARD, Pierre, Aspects du Nouvel-Ontario au XIXe siècle, 36, 2: 263; Pierre BOGLIONI et Benoît LACROIX, dir., Les pèlerinages au Québec, 39, 3: 440-441; Gérard BOISMENU, Laurent MAILHOT et Jacques ROUILLARD, Le Québec en textes, 1940-1980, 35, 2: 273-274; Luigi BRUTI-LIBERATI, Il Canada, L'Italia e il fascismo (1919-1945), 40, 1: 116; Gaston CARRIÈRE, Dictionnaire biographique des Oblats de Marie-Immaculée au Canada, tome 1, 31, 2: 259-260; André CORVISIER, Arts et sociétés dans l'Europe XVIIIe siècle, 33, 1: 81-82; Lionel DORGE, Le Manitoba, reflets d' un passé, 31, 1: 96-97; Gabriel DUSSAULT et Gilles MARTEL, Charisme et économie. Les cinq premières communautés masculines établies au Québec sous le Régime anglais (1837-1870), 39, 2: 283-284; Maurice FILION, dir., Hommage à Lionel Groulx, 31, 4: 575-576; André LATREILLE, De Gaulle, la Libération et l'Église catholique, 33, 1: 92-93; Jim LOTZ et William METCALFE, ed., Understanding Canada. A Multidisciplinary Introduction to Canadian Studies, 37, 3: 465-466; Manoly R. LUPUL, ed., A Heritage in Transition: Essays in the History of Ukrainians in Canada, 37, 4: 623-624; Laurent MAILHOT et Pierre NEPVEU, La poésie québécoise des origines à nos jours. Anthologie, 37, 2: 353-354; Émile POULAT, Église contre Bourgeoisie. Introduction au devoir du catholicisme actuel, 32, 1: 104-105; Émile POULAT, Catholicisme, démocratie et Socialisme. Le mouvement catholique et Mgr Benigni de la naissance 
du socialisme à la victoire du fascisme, 32 , 1: 104-105; Guildo ROUSSEAU, L'image des États-Unis dans la littérature québécoise (1775-1930), 36, 1: 123-126

SEAGER, Frédéric, Irving ABELLA et Harold TROPER, None is Too Many: Canada and the Jews of Europe, 1933-1948, 39, 2: 271272

SÉGUIN, Normand, Christian MORISSONNEAU, La terre promise: le mythe du Nord québécois, 33, 3: 473-476

SENESE, Phyllis M., Doug OWRAM, The Government Generation: Canadian Intellectuals and the State 1900-1945, 40, 4: 605608

SIMARD, Jean-Jacques, Micheline DUMONT, Michèle JEAN, Marie LAVIGNE et Jennifer STODDART, L'histoire des femmes au Québec depuis quatre siècles, 38, 1: 101

SIMARD, Sylvain, Serge GAGNON, Le Québec et ses historiens, de 1840 à 1920 - La Nouvelle-France de Garneau à Groulx, 35, 1: 104-106; John R. PORTER, Joseph Légaré 1795-1855 - L'oeuvre, 33, 2: 273276

SMITH, Donald B., P.-André SÉVIGNY, Les Abénaquis: Habitat et migrations (17e et 18e siècles), 31, 4: 589-591

SOLDATOS, P., Edmond ORBAN, Un modèle de souveraineté-association? Le Conseil nordique, 33, 2: 270-272

SOUTHAM, Peter, Alvin FINKEL, Business and Social Reform in the Thirties, 34, 1: 110-112; David KAREL, Jean SIMARD et Elzéar LAVOIE, dir., L'Interdisciplinarité au Département d' histoire. Actes de colloque, 35, 2: 291; «M. Esdras Minville: 1896-1975», L'Action nationale, 31, 2: 268-269

SPIRIDONAKIS, B. G., Aloysius BALAWYDER, ed., Canadian-Soviet Relations, 1939-1980, 37, 1: 91-92

STEWART, Michel, Brian J. YOUNG, Promoters and Politicians: the North-Shore Railways in the History of Quebec, 1854-85, 33, 1: 101-103

STODDART, Jennifer, Hugh ARMSTRONG et Pat ARMSTRONG, The Double Ghetto: Canadian Women and their Segregated Work, 34, 1: 99-100; R. G. MOYLES, The Blood and Fire in Canada: A History of the
Salvation Army in the Dominion, 18821976, 32, 2: 267-270

SWEENY, Robert, Peter C. NEWMAN, L'establishment canadien - Ceux qui détiennent le pouvoir, 37, 3: 468-470

SYLVAIN, Philippe, Nadia F. EID, Le clergé et le pouvoir politique au Québec. Une analyse de l'idéologie ultramontaine au milieu du XIXe siècle, 34, 1: 105-110

$\mathbf{T}$

TASCHEREAU, Sylvie, Karl AUN, The Political Refugees: a History of the Estonians in Canada, 40, 1: 106-109; Milda DANYS, DP: Lithuanian Immigration to Canada After the Second World War, 40, 4: 613-615 TESSIER, Daniel, Réjean OLIVIER, Catalogue de laurentiana, de canadiana et de livres anciens de la famille Olivier, répertoriant 12000 volumes et 120 collections de périodiques, pour la plupart du Québec, 39, 2: 294-296

TÉTREAULT, Martin, Colloques de l'Institut français du collège de l'Assomption, 39, 2: 277-281

THÉRIAULT, Michel, Cornelius JAENEN, The Role of the Church in New France, 31, 1: 100-103

TOUSIGNANT, Pierre, Fernand OUELLET, Le Bas-Canada, 1791-1840: «Le Bas-Canada: une étape importante dans l'oeuvre de Fernand Ouellet» (NC), 34, 3: 415-436

TREMBLAY, Marc-Adélard, Lauraine LÉGER, Les sanctions populaires en Acadie, 33, 1: 93-95

TRÉPANIER, Plerre, Arthur BUIES, Chroniques, tome 1, éd. critique par Francis PARMENTIER, 40, 4: 598-600; F. DUMONT, J. HAMELIN et J.-P. MONTMYNY, dir., Idéologies au Canada français, 1930-1939, 35, 1: 101-104; Cyrille FELTEAU, Histoire de La Presse, tome I: Le livre du peuple, 1884-1916; tome 2: Le plus grand quotidien français d'Amérique, 1916-1984, 39, 1: 98-99; R. Douglas FRANCIS, Frank $H$. Underhill: Intellectual Provocateur, 40, 3: 437-439; Lionel 
GROULX, Journal, 1895-1911, éd. critique par Giselle HUOT et Réjean BERGERON, 39, 2: 286-289; Gilles MARTEL, Le messianisme de Louis Riel, 39, 1: 107-109; Robert RUMILLY, L'Acadie anglaise (1713-1755), 37, 4: 629-631; Robert RUMILLY, L'Acadie française (14971713), 36, 3: 439-440; André VACHON, La Famille Drouin au Perche, 1551-1636, étudiée d'après des documents découverts et relevés par Madame Pierre Montagne, 40, 1: 112-113

TROFIMENKOFF, Susan Mann, Denise LEMIEUX et Lucie MERCIER, $L a$ recherche sur les femmes au Québec. Bilan et bibliographie, 37, 1: 118-119

TRUDEL, François, Frederick W. ROWE, Extinction: The Beothuks of Newfoundland, 32, 4: 640-642

TRUDEL, Marcel, Lucien CAMPEAU, Monumenta Novae Franciae, vol. II: Établissement à Québec (1616-1634), 36, 4: 584586; André CHARBONNEAU, Yvon DESLOGES et Marc LAFRANCE, Québec, ville fortifiée du XVIIe siècle au XIXe siècle, 37, 2: 329-331; François-Marc GAGNON et Denise PÉTEL, Hommes effarables et bestes sauvaiges. Images du NouveauMonde d'après les voyages de Jacques Cartier, 40, 4: 583-588; Dom Guy-Marie OURY, Jeanne Mance et le rêve de M. de La Dauversière, 38, 1: 111-113; Jean-Marc SOYEZ, Quand l'Amérique s'appelait Nouvelle-France, 1608-1760, 36, 2: 277-278
101-103; D. K. FOOT, Provincial Public Finance: an Empirical Analysis of the Last Twenty-Five Years, 33, 4: 590-592; James Iain GOW, Histoire de l'administration publique québécoise, 1867-1970, 40, 3: 432-434; Yvan LAMONDE, Guide d'histoire du Québec, 32, 2: 265-266; Dianne NEWELL, Technology on the Frontier: Mining in Old Ontario, 40, 4: 593-595

VÉRONNEAU, Pierre, Pierre-François HÉBERT et Yvan LAMONDE, Le cinéma au Québec. Essai de statistique historique (1896 à nos jours), 38, 2: 265-267

VÉZINA, Raymond, Fernand LEDUC, Vers les îles de lumière. Écrits (1942-1980), 36, 4: 593-595; Dennis REID, "Notre patrie le Canada». Mémoires sur les aspirations nationales des principaux paysagistes de Montréal et de Toronto 1860-1890, 36, 1: 120-123

VOISINE, Nive, Léon BÉLANGER, L'Islet, 1677-1977, 34, 1: 100; André CHAPEAU, Louis-Philippe NORMAND et Lucienne PLANTE, Évêques catholiques du Canadal Canadian R. C. Bishops, 1658-1979, 36, 1: 109-110; Pierre CHARBONNEAU, Le projet québécois d'Honoré Mercier, 37, 1: 118; Luca CODIGNOLA, Vatican: Archives de la Sacrée Congrégation de la Propagande, 38, 4: 592-593; Gabriel DUSSAULT, Le Curé Labelle. Messianisme, utopie et colonisation au Québec (1850-1900), 38, 4: 593-594; Jacques GRISÉ, Les conciles provinciaux de Québec et l'Église canadienne (1851-1886), 33, 4: 594-596; Benoît LACROIX, Folklore de la mer et religion, 35, 1: 118; Michel LANGLOIS, Cherchons nos ancêtres, 35, 2: 291-292; André VACHON, François de Laval, 35, 3: 433434

VACHET, André, Nicole LAURIN-FRENETTE, Production de l'État et formes de la nation, 34, 3: 444-448

VACHON, André, Paul AUBIN, Bibliographie de l'histoire du Québec et du Canada, 1966-1975, 38, 3: 449-450

VALLIĖRES, Gaétan, René BRODEUR et Robert CHOQUETTE, Villages et visages de l'Ontario français, 35, 3: 411-413

VALLIĖRES, Marc, Judith FINGARD, Jack in Port: Sailortowns of Eastern Canada, 38, 1 :

\section{W}

WAITE, P. B., C. P. STACEY, Canada and the Age of Conflict: A History of Canadian External Policies, vol. 1: 1867-1921, 33, 2: 276-278 


\section{Revue d'histoire de l'Amérique française}

WATELET, Hubert, René HARDY et Serge GAGNON, dir., L'Église et le village au Québec 1850-1930. L'enseignement des Cahiers de prônes. Textes de André Audet, Guy Trépanier, Carmen Rousseau, 35, 4: 594-596

WEAVER, John C., Paul-André LINTEAU, Maisonneuve. Comment des promoteurs fabriquent une ville, 36, 1: 112-118

WHITELEY, W. H., Kenneth JOHNSTONE, The Aquatic Explorers: a History of the Fisheries Research Board of Canada, 33, 3: 465467

WILLIS, John, H. Clare PENTLAND, Labour and Capital in Canada 1650-1860 (NC), 38, 2: 245-253

YOUNG, Brian, Alan F. ARTIBISE et Gilbert A. STELTER, eds., The Canadian City: Essays in Urban History, 32, 1: 111-113

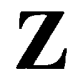

ZOLTVANY, Yves F., André VACHON, avec la collaboration de Victorin CHABOT et d'André DESROSIERS, Rêves d'empires: le Canada avant 1700, 37, 4: 635-636 